Article

\title{
Uniform and Robust Peptoid Microsphere Coatings
}

\section{Melissa L. Hebert, Dhaval S. Shah, Phillip Blake and Shannon L. Servoss *}

Ralph E. Martin Department of Chemical Engineering, University of Arkansas, 3202 Bell Engineering Center, Fayetteville, AR 72701, USA; E-Mails: melissa.hebert2@gmail.com (M.L.H.); dshah@uark.edu (D.S.S.); blake.p.a@gmail.com (P.B.)

* Author to whom correspondence should be addressed; E-Mail: sservoss@uark.edu; Tel.: +1-479-575-4502.

Received: 21 May 2013; in revised form: 13 June 2013 / Accepted: 14 June 2013 /

Published: 21 June 2013

\begin{abstract}
Peptoids that are helical and partially water soluble have been shown to self-assemble into microspheres when the peptoid solution is dried on a silicon substrate. Such microsphere coatings have great potential for use in biosensor technologies, specifically to increase the surface area for binding. However, in order to be useful, the peptoids must consistently form uniform coatings. In this study we investigated the effects of various coating protocol parameters on the uniformity of the resulting peptoid microsphere coatings, including (i) solvent, (ii) administration technique, and (iii) drying environment. In addition, we investigated the robustness of the coatings as well as the potential for using a glass substrate. These studies show that uniform, robust peptoid microsphere coatings can be formed using protic solvents, a full coverage administration technique, and drying in open air on silicon or glass substrates.
\end{abstract}

Keywords: coating; biosensor; peptoid; microsphere

\section{Introduction}

The pursuit of an efficient, non-invasive, and economical tool for the early detection of complex diseases, such as cancer, has led to the development of sophisticated biosensor technologies for protein analysis. As biosensors become more complex, coatings become increasingly important. There are numerous concerns with existing coatings including low stability, non-specific binding, and potential for increased sensitivity. A coating is needed that has the following characteristics: (i) high surface 
area for binding, (ii) resistance to biofouling, and (iii) flexible synthesis that allows for incorporation of a variety of chemistries. Such a coating is possible using self-assembled poly-N-substituted glycine (or peptoid) microspheres.

Peptoids are biomimetic polymers with a backbone similar to that in proteins. In peptoids, the side chains are appended to the amide groups rather than the $\alpha$-carbons [1]. Although peptoids lack the ability to form backbone hydrogen bonds that induce a stable helical fold in proteins, it has been shown that peptoids with bulky chiral, aromatic side chains are able to form robust helical secondary structures [2-6] that are stabilized by steric interactions [7]. Stable helices can be formed by peptoids as short as five residues with circular dichroism (CD) spectra that strongly resemble those of protein $\alpha$-helices $[5,8]$. NMR studies show that peptoids have a helical structure similar to polyproline type-I helices in proteins, with a periodicity of three residues per turn and a pitch of $\sim 6 \AA$ [7].

Recently, carefully designed peptoids have been shown to self-assemble into various supramolecular assembly structures including superhelices [9], crystalline sheets [10], ribbons [11], monolayers that can further form into stable nanosheets [12], and microspheres [13]. Peptoids have great potential for use as biosensor coatings due to their ability to increase surface area through supramolecular assemblies, their ease of synthesis, and the numerous side chain chemistries available. Previous work in our lab has shown that peptoids that have helical secondary structure and are partially water soluble are able to self-assemble into microspheres [13]. The ability to form microspheres and the diameter of the microspheres can be tailored by varying the side chain placement and chemistry.

In this paper we investigate the effect of coating protocol on the uniformity of peptoid microsphere coatings. Specifically, solvent type, administration technique, and drying environment were investigated and changes in coating uniformity were monitored. We found that protic solvents, drying at standard conditions, and full coating of the slide reproducibly resulted in uniform coatings. Utilizing these protocols, the peptoid microsphere coatings are robust and can be created on glass and silicon substrates.

\section{Materials and Methods}

\subsection{Materials}

(S)-Methylbenzylamine was purchased from Acros Organics (Pittsburgh, PA) and tert-butyl $\mathrm{N}$-(4-aminobutyl)carbamate was purchased from $\mathrm{CNH}$ Technologies Inc. (Woburn, MA). $\mathrm{H}$-Alanine-OtBu$\cdot \mathrm{HCl}$ was purchased from BACHEM (Torrance, CA) and underwent a free-base reaction before use in peptoid synthesis. Acetic anhydride was purchased from Alfa Aesar (Ward Hill, $\mathrm{MA}$ ) and sequence grade $N, N$-diisopropylethylamine (DIEA) was purchased from Pierce (Rockford, IL). MBHA rink amide resin was purchased from NovaBiochem (Gibbstown, NJ). Test grade silicon wafers were purchased from University Wafer (South Boston, MA) and ultra clean glass microarray slides were purchased from Thermo Scientific (Pittsburgh, PA). All other reagents for peptoid synthesis, purification and sample preparation were purchased from VWR (Radnor, PA) and used without any further purification or modification. 


\subsection{Peptoid Synthesis and Purification}

Peptoids were synthesized via the submonomer addition method [1] on rink amide resin using a 433A Applied Biosystems Peptide Synthesizer (Carlsbad, CA) that was refurbished from a 431A Peptide Synthesizer. The N-terminus of peptoid A was acetylated in 50-fold molar excess acetic anhydride and $50 \mu \mathrm{M}$ ammonium bicarbonate as the final step of synthesis. Peptoids were cleaved from the resin by bathing in a solution of 95\% triflouroacetic acid (TFA), 2.5\% triisopropyl silane and $2.5 \%$ water for two to ten minutes. The acid was removed using a Heidolph Laborota 4001 rotating evaporator (Elk Grove Village, IL) and the samples were diluted to a concentration of $\sim 3 \mathrm{mg} / \mathrm{mL}$ in a 50:50 solution of acetonitrile:water. Peptoids were purified using a Waters Delta 600 preparative high performance liquid chromatography (HPLC) instrument (Milford, MA) with a Duragel G C18 $150 \times 20 \mathrm{~mm}$ column (Peeke Scientific, Novato, CA) and gradients of approximately 1\% per minute acetonitrile over a range that included the elution percentages for each peptoid. These gradients were created using solvents $\mathrm{A}$ and $\mathrm{B}$, where solvent $\mathrm{A}$ is $95 \%$ water, $5 \%$ acetonitrile, $0.1 \%$ TFA and solvent $\mathrm{B}$ is $95 \%$ acetonitrile, $5 \%$ water, $0.1 \%$ TFA. Peptoids were confirmed to be $>97 \%$ pure via reversed-phase analytical HPLC (Waters Alliance, Milford, MA) using a Duragel G C18 $150 \times 2.1 \mathrm{~mm}$ column (Peeke Scientific, Novato, CA) and a linear gradient of 5 to $95 \%$ solvent D (acetonitrile, $0.1 \%$ TFA) in solvent $\mathrm{C}$ (water, $0.1 \%$ TFA) over $30 \mathrm{~min}$. The purified molecular weights were confirmed to match theoretical values using matrix-assisted laser desorption/ionization (MALDI) mass spectrometry. A-1866 Da, 83.8\% HPLC elution in acetonitrile; B-1920 Da, 82.4\% HPLC elution in acetonitrile. Purified peptoid solutions were dried to a powder using a Labconco lyophilizer (Kansas City, MO, USA).

\subsection{Sample Preparation}

The dried peptoids were dissolved in a solution of 4:1 organic:water, where the organic is methanol, ethanol, isopropanol, or acetonitrile at a concentration of $3 \mathrm{mg} / \mathrm{mL}$. Peptoid solutions were dried onto silicon wafer chips $(\sim 1 \mathrm{~cm} \times 1 \mathrm{~cm})$ using various combinations of administration technique and drying environment. The three administration techniques that were investigated include: (i) pipette spotsmall sample volume ( $\sim 5 \mu \mathrm{L})$ administered to the chip using a pipette, (ii) full coverage-large sample volume $(20-50 \mu \mathrm{L})$ administered to the chip using a pipette and fully coating the surface, and (iii) dip — submerging the chip in the sample and removing it in a vertical orientation. The three drying environments that were investigated include: (i) open air - chips were placed in an open Petri dish and dried at standard room conditions for $\sim 1$ hour, (ii) humidity chamber-chips were placed in a closed container controlled to $60 \%$ humidity for 3-4 hours, and (iii) vacuum chamber - chips were placed in a container and house vacuum was applied for $\sim 5$ minutes.

\subsection{Scanning Electron Microscopy}

The dried peptoid coatings were visually analyzed using a Phillips XL-30 environmental scanning electron microscope (ESEM) to observe surface morphology and determine the effect of the given conditions to form a uniform self-assembled peptoid microsphere coating on the substrate. 


\section{Results and Discussion}

\subsection{Peptoid Sequence Design and Rationale}

Previous studies in our lab have shown that helical peptoids that are partially soluble in water form microspheres when dried from aqueous/organic solutions [13]. While these studies showed that the peptoids were able to reproducibly form microspheres, the coatings were not always uniform. Here we chose to use the two peptoids from the previous study that most consistently formed microsphere coatings. For simplicity, we will refer to the peptoids as $\mathbf{A}$ and $\mathbf{B}$ (structures shown in Figure 1). $\mathbf{A}$ and B both contain two helical faces composed of chiral, aromatic functional groups that induce secondary helical structure [2-6]. The third helical face contains either negatively and positively charged groups (A) or positively charged and aromatic ether groups (B). In both cases, self-assembly likely occurs due to aromatic stacking and hydrophobic effects. Interestingly, A forms microspheres nearly 10 times smaller than $\mathbf{B}(\mathbf{A}=0.34 \pm 0.19 \mu \mathrm{m}$ and $\mathbf{B}=3.2 \pm 1.6 \mu \mathrm{m})$ [13]. This is likely due to the ability of the negatively and positively charged groups to interact to form a tighter supramolecular assembly.

Figure 1. Chemical structures for peptoids A and B.

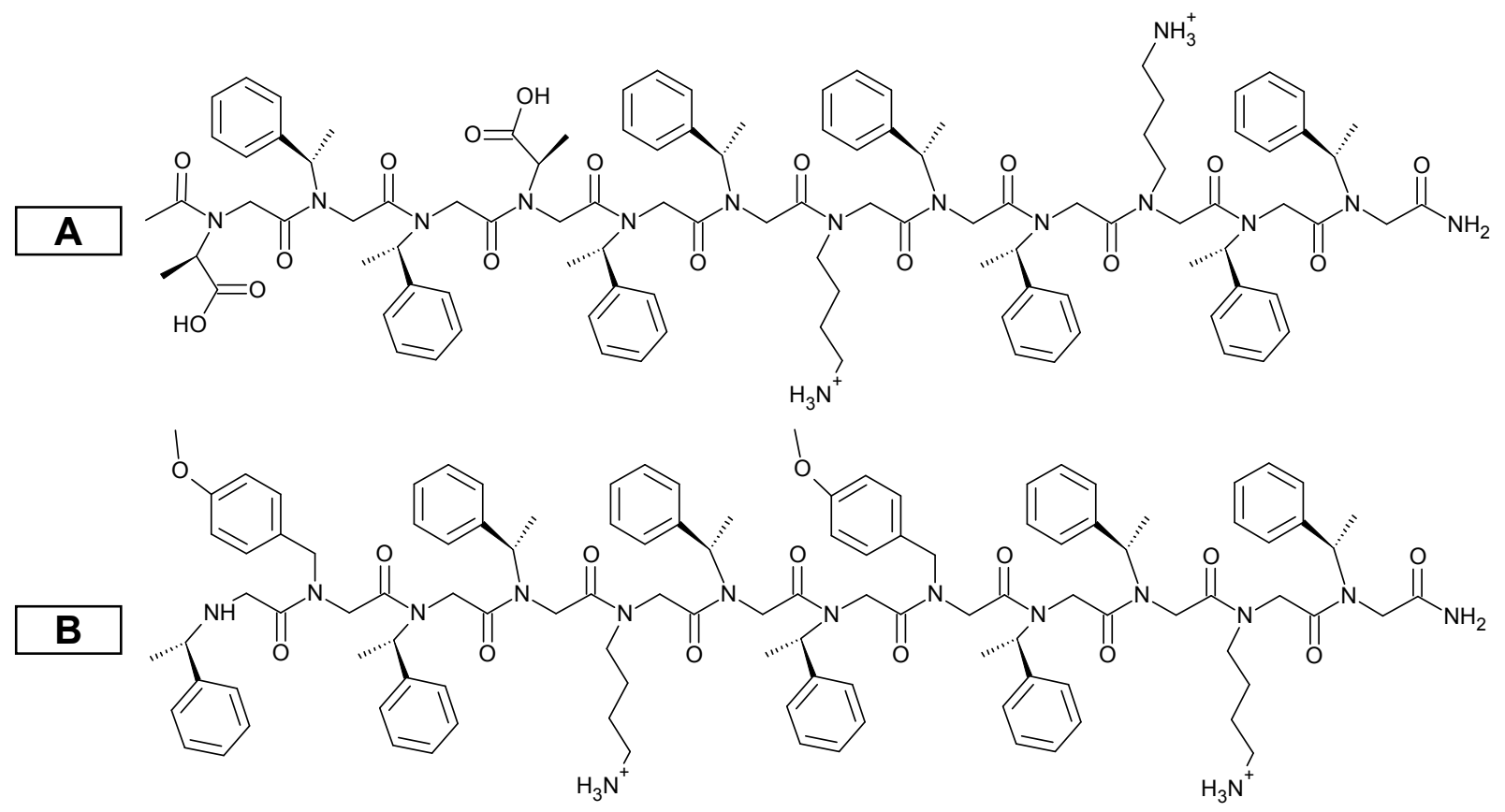

\subsection{Solvent}

The importance of solvent choice was investigated using three protic solvents and one non-protic solvent. Protic solvents have been shown to help stabilize the secondary helical structure of peptoids through hydrogen bonding [14]. Peptoids were dissolved in 4:1 organic:water solutions: (i) methanol, (ii) ethanol, (iii) isopropanol, and (iv) acetonitrile. All samples were administered by full coverage and dried in open air.

SEM images show that peptoid microsphere coatings form in all of the protic solvents for both $\mathbf{A}$ and $\mathbf{B}$ (Figure $2 \mathrm{~A}-\mathrm{C}, \mathrm{E}-\mathrm{G}$ ). While uniform coatings were observed for isopropanol and ethanol, the methanol coatings were spotty under these conditions. A did self-assemble into microspheres in the 
non-protic solvent acetonitrile, but the coatings were very spotty (Figure 2D). B did not form microspheres at all in the presence of acetonitrile (Figure $2 \mathrm{H}$ ). These results suggest that the use of any protic solvent will allow for the uniform formation of peptoid microsphere coatings.

Figure 2. SEM images for $\mathbf{A}(\mathrm{A}-\mathrm{D})$ and $\mathbf{B}(\mathrm{E}-\mathrm{H})$. Peptoids were dissolved in 4:1 organic:water, administered by full coverage, and dried under open air conditions. Organic is methanol ( $\mathrm{A}$ and $\mathrm{E}$ ), ethanol ( $\mathrm{B}$ and $\mathrm{F}$ ), isopropanol ( $\mathrm{C}$ and $\mathrm{G}$ ), and acetonitrile (D and $\mathrm{H})$. Scale bars are as indicated.

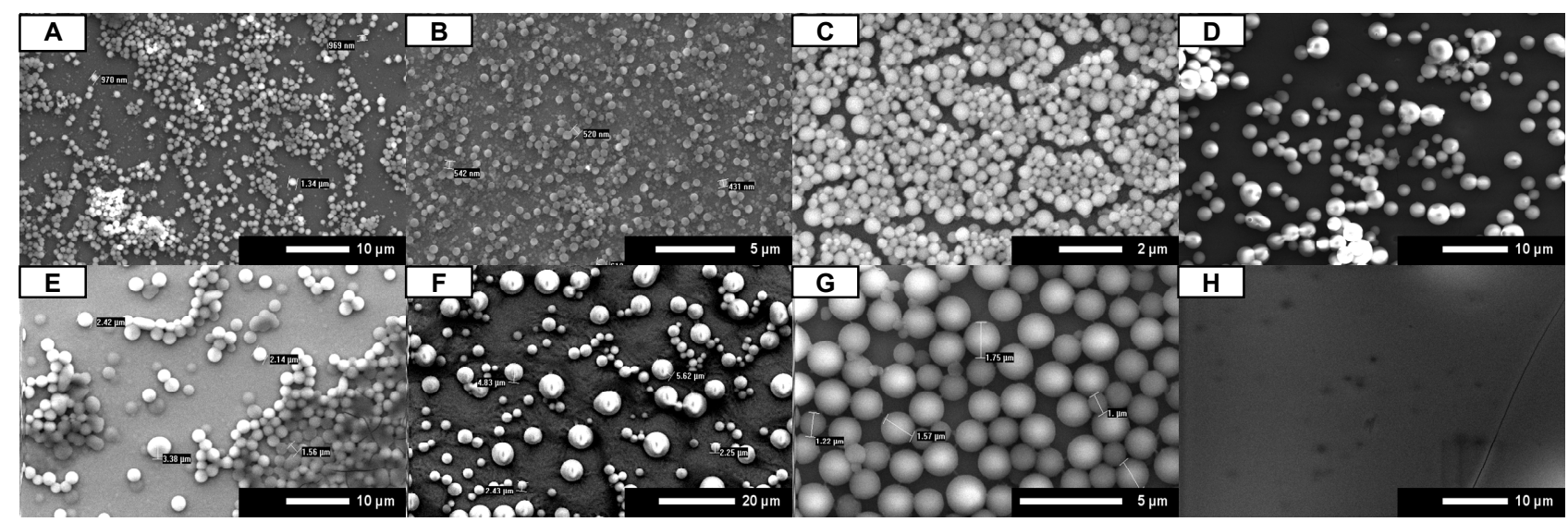

\subsection{Administration Technique}

Another parameter that can have importance in the formation of uniform microspheres is the administration technique. Coatings can be administered very simply by pipette or by methods that are more readily adapted to a high throughput protocol, such as dipping. Here we utilized three administration techniques to determine the effect on coating uniformity: (i) pipette spot, (ii) full coverage, and (iii) dip. For each administration technique, the peptoid was dissolved in 4:1 isopropanol:water and allowed to evaporate from the slide at standard room conditions.

SEM images (Figure 3) clearly show that the administration technique has a large effect on the uniformity of the microsphere coating for both $\mathbf{A}$ and $\mathbf{B}$. While both pipette spot (Figure 3A,D) and full coverage (Figure 3B,E) techniques resulted in the formation of microspheres, only the full coverage technique consistently resulted in uniform slide coatings. When the chip was dipped in the peptoid solution, no distinct microspheres were formed (Figure 3C,F). This indicates that the peptoid microspheres do not adhere to the substrate until the solvent begins to evaporate from the surface. This conclusion was further confirmed by an extended dip method experiment where a small silicon wafer chip was submerged in a peptoid solution (A at $3 \mathrm{mg} / \mathrm{mL}$ in 4:1 ethanol:water) for five minutes. The silicon chip was removed from the peptoid solution in a vertical orientation and allowed to dry in open air. As with the original dip method experiments, there was no formation of uniform microsphere coatings observed on the substrate following the extended submersion time (data not shown). These results suggest that the substrate must be uniformly covered with the peptoid solution for uniform microsphere coatings to form. 
Figure 3. SEM images for $\mathbf{A}(\mathrm{A}-\mathrm{C})$ and $\mathbf{B}(\mathrm{D}-\mathrm{F})$. Peptoids were dissolved in 4:1 isopropanol:water and dried under open air conditions. Peptoids were administered by pipette spot (A and D), full coverage (B and E), and dipping (C and F). Scale bars are as indicated.

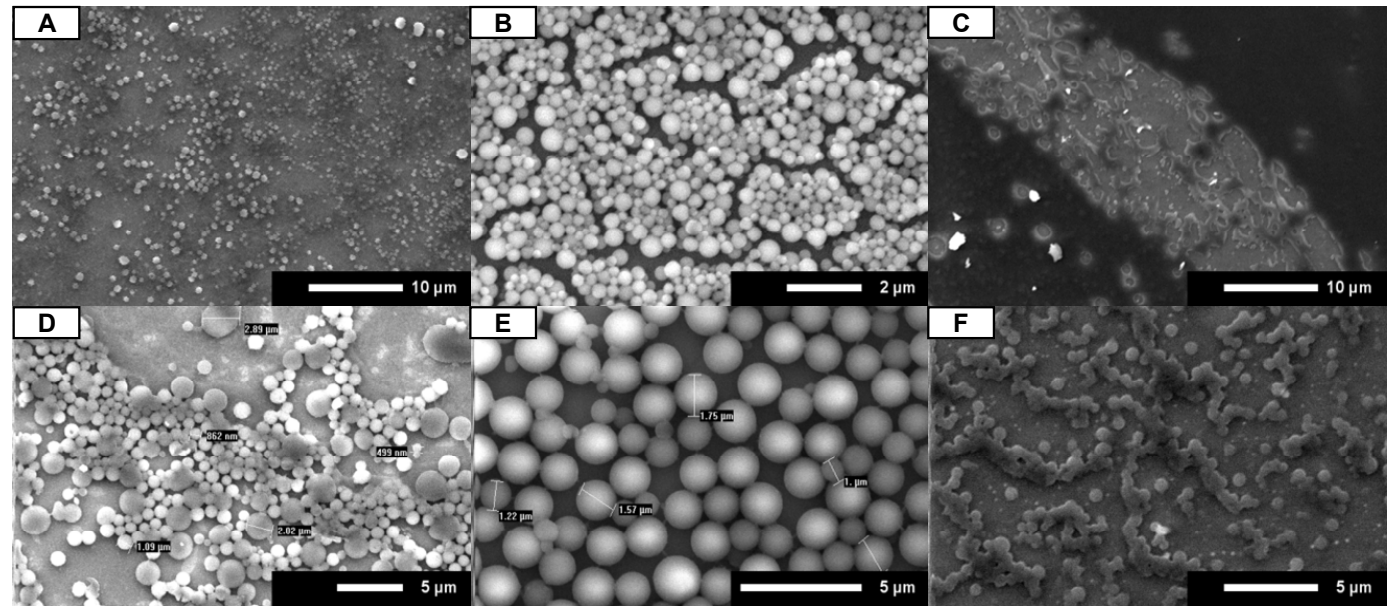

\subsection{Drying Environment}

The effect of drying environment, and therefore drying time, was investigated by adjusting the humidity during evaporation. Humidity and vacuum chambers were used to create slow and quick drying environments. The conditions for the evaporation step were (i) open air-about $1 \mathrm{~h}$, (ii) humidity chamber-3-4 h at 60\% humidity, and (iii) vacuum chamber-about 5 min.

Figure 4. SEM images for $\mathbf{A}(\mathrm{A}-\mathrm{C})$ and $\mathbf{B}$ (D-F). Peptoids were dissolved in 4:1 isopropanol:water and administered by full coverage. Peptoids were dried in open air (A and D), humidity chamber (B and E), and vacuum chamber (C and F). Scale bars are as indicated.

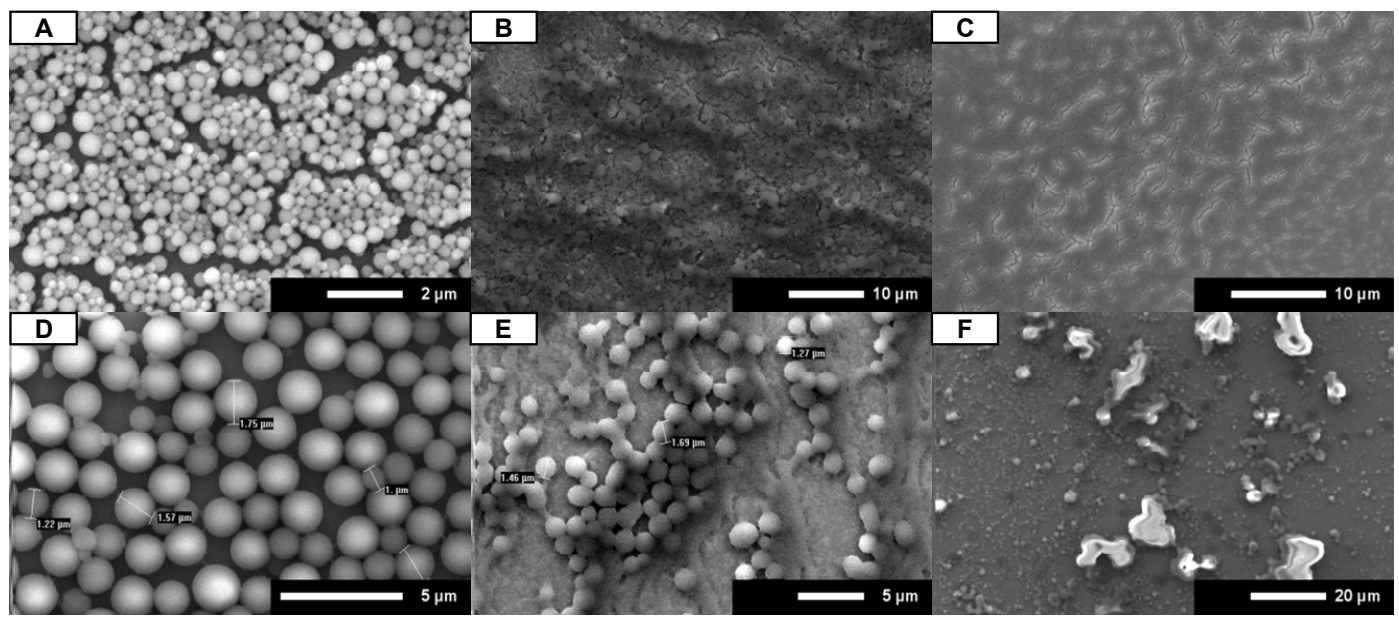

When dried in open air conditions, both $\mathbf{A}$ and $\mathbf{B}$ consistently formed uniform microsphere coatings (Figure 4A,D). Both slow drying and fast drying resulted in poor formation of uniform microsphere coatings. When $\mathbf{A}$ and $\mathbf{B}$ were allowed to dry in a humidity chamber they were observed to start drying at the outer edges of the chip and finish in the center, as indicated by increased microsphere density at 
the center of the chip (Figure 4B,E). Quick drying of A and B in a vacuum chamber resulted in spotty microsphere coatings or a complete lack of microspheres (Figure 4C,F). The microspheres imaged after vacuum drying were much smaller than others observed and were not spherical. These results suggest that drying at standard conditions results in uniform microsphere coatings.

\subsection{Coating Robustness and Versatility}

Testing of coating protocol parameters has shown that uniform peptoid microsphere coatings can be consistently formed. In order to be useful in biosensor applications, the coatings must be robust through multiple washing steps and conditions, as well as form on various substrate chemistries. Here we investigate robustness through repeated washing with phosphate-buffered saline/Tween $(0.05 \%)$ and deionized water.

Minimal changes in the uniformity of the coating were observed, as shown in Figure 5A,D, following repeated wash steps in high salt and surfactant. Image J analysis of the peptoid microsphere coating before and after the washes showed that the slide area covered by peptoid microspheres had only a slight decrease, from $19.6 \%$ to $18 \%$, of total slide area. At a higher magnification of the coatings, the peptoid microspheres appear to be hollowed out or wrinkled on the surface (Figure 5B,E), likely due to interactions between the peptoid and the surfactant (Tween). This was tested by treating the slide with a blocking agent (casein) prior to the wash steps, which is a standard step in protein assays and biosensor devices to prevent non-specific binding to the substrate. As observed in Figure 5C,F, coating with a blocking agent prevents the hollowing or wrinkling of the peptoid microspheres.

Figure 5. SEM images before $(A-C)$ and after $(D-F)$ washing. Coatings are $\mathbf{B}$ in 4:1 ethanol:water, administered by full coverage, and dried in open air. The coatings shown in panels $\mathrm{C}$ and $\mathrm{F}$ were treated with casein prior to washing. Scale bars are as indicated.

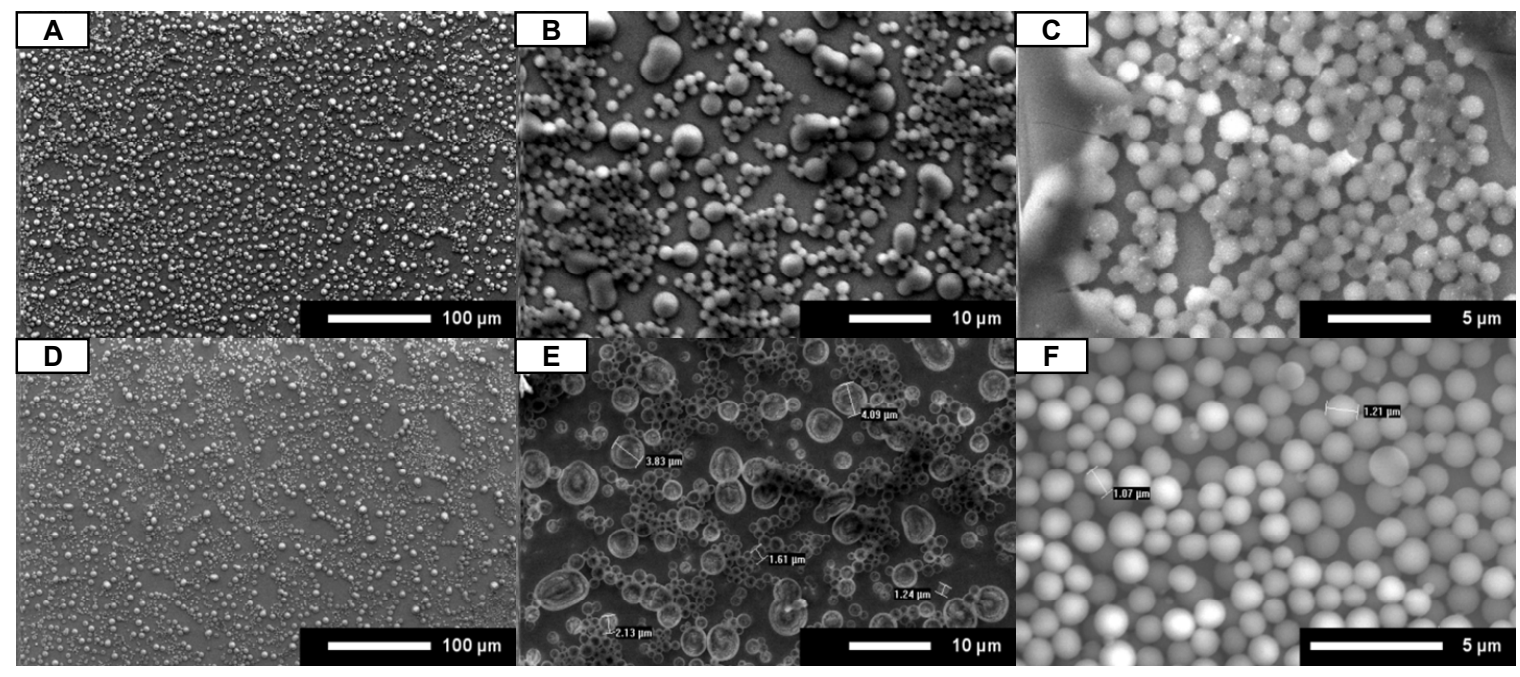

The ability to form uniform peptoid microsphere coatings on other substrates was investigated using ultraclean glass slides that are commonly used in ELISA microarray assays. Peptoid was dried onto a glass slide using the best conditions as determined in our lab (protic solvent, full coverage, and open air drying). The slides were imaged with no further processing, resulting in blurry images due to 
the low conductivity of the glass substrate. However, as shown in Figure 6, a uniform coating of peptoid microspheres did form.

Figure 6. SEM image of uniform surface coating on a glass slide. Coating is $\mathbf{B}$ in 4:1 isopropanol:water, administered by full coverage, and open air drying. Scale bar is $5 \mu \mathrm{m}$.

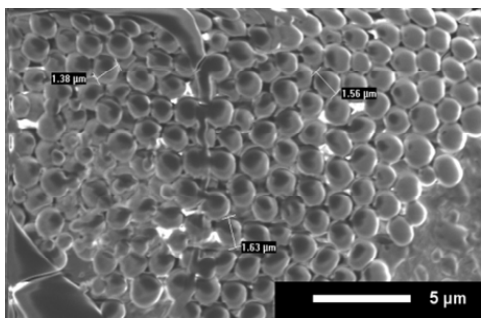

\section{Conclusions}

To our knowledge, this is the first thorough investigation of coating protocol for peptoid microspheres. Previous work from our lab showed that helical peptoids with partial water solubility reproducibly self-assemble into microspheres, but uniform coatings were not consistently achieved [13]. In this study the formation of uniform coatings was achieved by controlling the coating procedure. We investigated the use of various solvents, administration techniques, and drying environments to determine a protocol that resulted in the reproducible formation of uniform peptoid microsphere coatings.

While there was no difference observed in the formation of microspheres with the three protic solvents investigated (methanol, ethanol, and isopropanol), a non-protic solvent (acetonitrile) did not form uniform coatings. Since protic solvents have been shown to induce helical structure in peptoids, we believe any protic solvent will allow for the formation of uniform peptoid microsphere coatings. Both the full coverage and pipette spot techniques resulted in uniform coatings in the center of the slide, but coating from the pipetted spot technique had spotty areas near the edges of the spot that occurred due to drying effects. The dipped slides (both rapid and extended) did not result in uniform microsphere coatings. We believe this is because the peptoid microspheres do not adhere to the slide until the solvent begins to evaporate. The drying environment greatly affected the ability to form microspheres, as well as the uniformity of the coating. Drying the sample in a vacuum chamber resulted in small globular structures rather than microspheres. Drying in a humidity chamber resulted in high microsphere density at the center of the chip, with the microspheres clumping together in chains. Drying at standard conditions consistently resulted in the formation of uniform microsphere coatings. Further investigation of the peptoid microsphere coatings showed that they were quite robust when exposed to high salt conditions in the presence of surfactant and they were able to form on glass substrates.

These studies show that using a protic solvent, administering the peptoid using the full coverage technique, and drying in open air allows for the most uniform and robust coating of self-assembled peptoid microspheres on silicon or glass substrates. These coating protocol results are promising as they show standard processes used for biosensor technologies can be applied to peptoid microsphere coatings with no special considerations. 


\section{Acknowledgements}

The authors would like to thank Jeremiah Born for his assistance in the lab and Modi Wetzler for his intellectual input. The authors would like to acknowledge Suresh Kumar for use of and assistance with circular dichroism, the University of Arkansas Electron Optics Facility for use of scanning electron microscopy, and The Arkansas Statewide Mass Spectrometry Facility for use of mass spectrometry equipment. This work was supported by the Arkansas Biosciences Institute and University of Arkansas Start-Up funds.

\section{Conflict of Interest}

The authors declare no conflict of interest.

\section{References}

1. Zuckermann, R.N.; Kerr, J.M.; Kent, S.B.; Moos, W.H. Efficient method for the preparation of peptoids [oligo-( $N$-subsituted glycines)] by submonomer solid-phase synthesis. J. Am. Chem. Soc. 1992, 114, 10646-10647.

2. Armand, P.; Kirshenbaum, K.; Falicov, A.; Dunbrack, R.L., Jr.; Dill, K.A.; Zuckermann, R.N.; Cohen, F.E. Chiral $N$-substituted glycines can form stable helical conformations. Fold. Des. 1997, 2, 369-375.

3. Kirshenbaum, K.; Barron, A.E.; Goldsmith, R.A.; Armand, P.; Bradley, E.K.; Truong, K.T.; Dill, K.A.; Cohen, F.E.; Zuckermann, R.N. Sequence-specific polypeptoids: a diverse family of heteropolymers with stable secondary structure. Proc. Natl. Acad. Sci. USA 1998, 95, 4303-4308.

4. Wu, C.W.; Sanborn, T.J.; Huang, K.; Zuckermann, R.N.; Barron, A.E. Peptoid oligomers with alpha-chiral, aromatic side chains: sequence requirements for the formation of stable peptoid helices. J. Am. Chem. Soc. 2001, 123, 6778-6784.

5. Wu, C.W.; Sanborn, T.J.; Zuckermann, R.N.; Barron, A.E. Peptoid oligomers with alpha-chiral, aromatic side chains: effects of chain length on secondary structure. J. Am. Chem. Soc. 2001, 123, 2958-2963.

6. Sanborn, T.J.; Wu, C.W.; Zuckermann, R.N.; Barron, A.E. Extreme stability of helices formed by water-soluble poly- $N$-substituted glycines (polypeptoids) with alpha-chiral side chains. Biopolymers 2002, 63, 12-20.

7. Armand, P.; Kirshenbaum, K.; Goldsmith, R.A.; Farr-Jones, S.; Barron, A.E.; Truong, K.T.; Dill, K.A.; Mierke, D.F.; Cohen, F.E.; Zuckermann, R.N.; Bradley, E.K. NMR determination of the major solution conformation of a peptoid pentamer with chiral side chains. Proc. Natl. Acad. Sci. USA 1998, 95, 4309-4314.

8. Armand, P.; Kirshenbaum, K.; Falicov, A.; Dunbrack, R.L., Jr.; Dill, K.A.; Zuckermann, R.N.; Cohen, F.E. Chiral N-substituted glycines can form stable helical conformations. Fold. Des. 1997, 2, 369-375.

9. Murnen, H.K.; Rosales, A.M.; Jaworski, J.N.; Segalman, R.A.; Zuckermann, R.N. Hierarchical self-assembly of a biomimetic diblock copolypeptoid into homochiral superhelices. J. Am. Chem. Soc. 2010, 132, 16112-16119. 
10. Nam, K.T.; Shelby, S.A.; Choi, P.H.; Marciel, A.B.; Chen, R.; Tan, L.; Chu, T.K.; Mesch, R.A.; Lee, B.C.; Connolly, M.D.; Kisielowski, C.; Zuckermann, R.N. Free-floating ultrathin twodimensional crystals from sequence-specific peptoid polymers. Nat. Mater. 2010, 9, 454-460.

11. Chen, X.; Ding, K.; Ayres, N. Investigation into fiber formation in N-alkyl urea peptoid oligomers and the synthesis of a water-soluble PEG/N-alkyl urea peptoid oligomer conjugate. Polym. Chem. 2011, 2, 2635-2642.

12. Sanii, B.; Kudirka, R.; Cho, A.; Venkateswaran, N.; Olivier, G.K.; Olson, A.M.; Tran, H.; Harada, R.M.; Tan, L.; Zuckermann, R.N. Shaken, not stirred: collapsing a peptoid monolayer to produce free-floating, stable nanosheets. J. Am. Chem. Soc. 2011, 133, 20808-20815.

13. Hebert, M.L.; Shah, D.; Blake, P.; Turner, J.P.; Servoss, S.L. Tunable peptoid microspheres: Effects of side chain chemistry and sequence. Org. Biomol. Chem. 2013, DOI:10.1039/C3OB40561C.

14. De Santis, E.; Hjelmgaard, T.; Caumes, C.; Faure, S.; Alexander, B.D.; Holder, S.J.; Siligardi, G.; Taillefumier, C.; Edwards, A.A. Effect of capping groups at the $\mathrm{N}$ - and C-termini on the conformational preference of alpha,beta-peptoids. Org. Biomol. Chem. 2012, 10, 1108-1122.

(C) 2013 by the authors; licensee MDPI, Basel, Switzerland. This article is an open access article distributed under the terms and conditions of the Creative Commons Attribution license (http://creativecommons.org/licenses/by/3.0/). 